Journal of Case Reports 2021;11(3):199-202

\title{
Secondary Malignant Melanoma of Stomach
}

\author{
Kuldeep Chaudhary, Swati Mishra, Vatsala Misra, Himalina Sangma \\ Department of Pathology, Moti Lal Nehru Medical College, Prayagraj, Uttar Pradesh, India.
}

\begin{abstract}
Corresponding Author:
Dr Swati Mishra

Email: drswatimishra456@gmail.com

This is an Open Access article distributed under the terms of the Creative Commons Attribution License (creativecommons.org/ licenses/by/3.0).
\end{abstract}

Received Accepted

Published

\begin{abstract}
Background: Melanoma is a malignant tumor of the melanoytes and preponderantly occurs in the skin. Malignant melanomas are well known aggressive tumors that often metastatsize to lymph nodes and gastointestinal tract with small bowel, large bowel and rectum being the most common sites. However, gastric metastasis is a rare entity. It can be either primary or metastatic. Case Report: We report here two cases of secondary malignant melanoma of stomach, one with known and other with unknown primary lesion of 55 year and 75 year old patients respectively. Conclusion: Melanoma metastasizing to stomach is inimitable entity and augur a poor prognosis.
\end{abstract}

Keywords: Gastrointestinal Neoplasms, Melanoma, Rectum, Skin Neoplasms, Stomach.

\section{Introduction}

Malignant melanoma are known to be aggressive tumors with frequent metastasis to lymph nodes and viscera. The gastrointestinal tract is one of the frequent sites of metastasis from melanoma [1]. However, gastrointestinal (GIT) melanomas with unknown primary source are relatively rare and are known to carry a better prognosis compared to cases with known primary lesion [2]. The most common sites of the metastasis in GIT are small bowel (35-67\%), colon (9-15\%), with stomach (5$7 \%$ ) being a rare entity [3]. In case of cutaneous melanoma, the most common sites of GIT metastasis are small intestine, colon and eventually stomach [4]. The GIT metastasis to stomach presages a poor prognosis with median survival of 4 to 6 months [5].

\section{Case Reports}

\section{Case 1}

A 55-year-old male presented to the outpatient department of Gastroenterology with the complains of recurrent vomiting, loss of appetite, abdominal fullness and generalized weakness for last 1 month. The patient also underwent a hip surgery 1 year back. CT abdomen revealed hepatomegaly with metastatic lesions in both lobes along with pulmonary and bony metastasis. In upper gastro-intestinal (GI) endoscopy, multiple nodules of varying sizes from $0.2 \mathrm{~mm}$ to $1 \mathrm{~cm}$ with a black ulcerated area were seen. His routine blood investigations were within normal limit. The biopsy from the stomach was sent to us for histopathological examination. Six, irregular, grey white, tiny pieces of tissue were received. Sections examined showed round to polygonal cells with hyperchromatic nuclei and prominent nucleoli lying in small groups, clusters and sheets in the lamina propria, encircling the crypts at places and infiltrating the intervening acini along with giant cells and golden-brown pigment in the cytoplasm. Further follow up could not be done as we lost the patient.

\section{Case 2}

A 75-year-old male, known case of diabetes mellitus presented to the outpatient department of Gastroenterology with abdominal discomfort, generalized weakness and anemia since 3 months 
for which upper GI endoscopy was done and lesions similar to above mentioned case were observed. When we examined the patients for primary lesion, we found a non-healing ulcer on right foot. The biopsy of the ulcer was sent for the histopathological examination. Single, irregular, grey white, skin covered pieces of tissue were received which measured $1.5 \times 1.5 \mathrm{~cm}$ in size. Outer surface showed two grey white nodules the cut surface of which were homogenous dark brown. The sections examined showed keratinized stratified squamous epithelium. Subepithelial zone revealed proliferation of atypical melanocytes both radially and vertically. These cells were pleomorphic, large in size, have high $\mathrm{N}: \mathrm{C}$ ratio, hyperchromatic and showed dense pigmentation. Shoulder phenomenon was also seen. The immunohistochemistry panel advised in the above two mentioned cases were HMB 45, S 100 and Melan A. IHC panel was positive for HMB 45, S-100 and Melan A

\section{Discussion}

Melanoma is a malignant tumor of melanocytes and primarily occurs in the skin. Malignant melanoma is an aggressive tumor with an unusual predilection for the gastrointestinal tract, stomach being the second most common site after small bowel $[6,7]$. Malignant melanoma that involves the GI tract is either primary or metastatic. Primary GI melanoma can arise in other GI mucosal sites, like oesophagus, small bowel, colon, rectum, and anus, in the absence of primary cutaneous melanoma [8] that are documented by immunohistochemical stains such as HMB-45 and S100 [9]. Diagnosis of primary melanomas of the GI tract are arduous to diagnose at an early stage and are therefore associated with a worse prognosis. The most common sites of metastases are the lymph nodes $(73.6 \%)$ and lungs $(71.3 \%)$, followed by the liver $(58.3 \%)$, brain $(54.6 \%)$, bone $(48.6 \%)$, and adrenal glands $(46.8 \%)$ [10]. Melanomas with an unknown primary has better prognosis compared to those

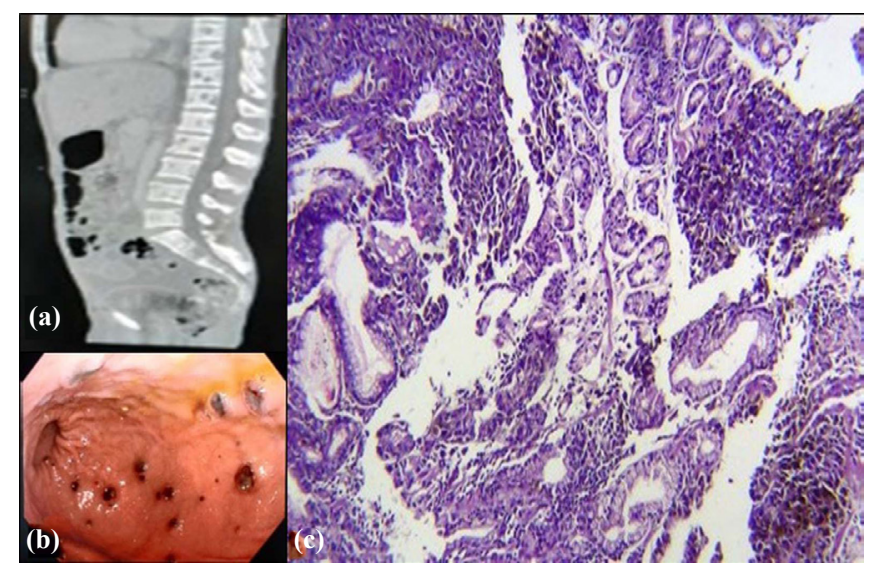

Fig.1: Clinico-pathological findings in case 1 (a): CT scan showing diffuse lytic bony lesions; (b): Multiple nodules with central ulcerated area on endoscopy; (c): Tumor showing round to polygonal cells in clusters and sheets, encircling pits ( $H$ and $E \times 10)$.

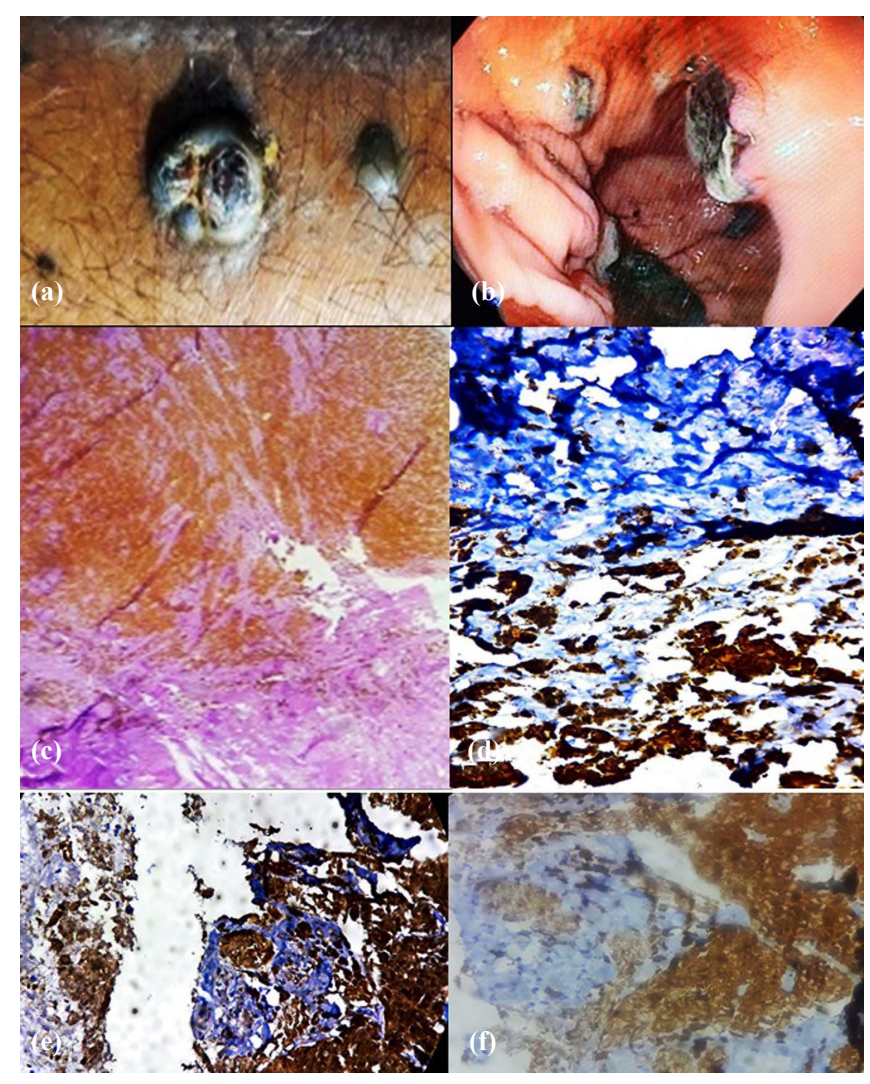

Fig.2: Clinico-pathological findings in case 2 (a): Nonhealing ulcer on foot; (b): Variable sized nodules with central black ulcerated area on endoscopy; (c): Atypical melanocytes with golden brown pigment in the cytoplasm ( $H$ and $E \times 10)$; (d): Tumor cells showing positivity for HMB $45(I H C \times 40)$; (e): Tumor cells showing positivity for $S 100$ (IHC $\times 40)$; (f): Tumor cells showing positivity for Melan A (IHC $\times 40)$. 
with known primary and are also relatively rare. Metastatic malignant melanoma with an unknown primary is a known entity with an incidence of $3.2 \%$ of all cases of malignant melanoma with a higher incidence in males and usually presenting in older age groups, mainly the fourth to fifth decade [11].

Diagnosis of metastatic melanoma is generally made by radiographic studies, that includes CT, ultrasonography, barium studies and endoscopic evaluation, through EGD, endoscopic retrograde cholangiopancreatography (ERCP) and colonoscopy. Recently, PET has been used to identify sites of metastatic melanoma [13]. The endoscopic classification of gastric metastases comprises three main morphological types [6]. First type includes melanotic nodules, often ulcerated at the tip. Second type comprises of submucosal tumor masses that are elevated and ulcerated at the apex. The last morphological type describes a mass lesion with varying incidence of necrosis and melanosis. The metastatic malignant melanoma upholds a poor prognosis. In various studies the mean survival of patients with systemic metastases from melanoma is ratified to be only 6 to 8 months [14]. Treatment recommended in patients of MUP (melanoma with unknown primary) includes surgery, immunotherapy, chemotherapy and radiation [15]. Surgical interventions performed are only for palliation of symptoms and improvement in survival [16].

\section{Conclusion}

Malignant melanoma of the GI tract is a challenging diagnostic entity demanding a thorough investigation for determination of its true primary lesion or metastasis. Our second case had metastatic melanoma from a regressed cutaneous growth on left leg. A detailed clinical and radiological examination is must to unveil the disease at an early stage for better prognosis and survival rate.
Contributors: KC, SM: manuscript writing, literature search; VM: manuscript editing, literature search; HS: critical inputs into the manuscript. KC will act as a study guarantor. All authors approved the final version of this manuscript and are responsible for all aspects of this study.

Funding: None; Competing interests: None stated.

\section{References}

1. Liang KV, Sanderson SO, Nowakowski GS, Arora AS. Metastatic malignant melanoma of the gastrointestinal tract. Mayo Clin Proc. 2006;81:511-516.

2. Vijuk G, Coates AS. Survival of patients with visceral metastatic melanoma from an occult primary lesion: A retrospective matched cohort study. Ann Oncol. 1998;9:419-422.

3. Pantalone D, Taruffi F, Paolucci R, Liguori P, Rastrelli M, Andreoli F. Malignant melanoma of the rectum. Eur J Surg. 2000;166:583-584.

4. Gupta TD, Brasfield R. Metastatic melanoma. A clinicopathological study. Cancer. 1964;17:1323-1339.

5. Liang KV, Sanderson SO, Nowakowski GS, Arora AS. Metastatic malignant melanoma of the gastrointestinal tract. Mayo Clinic Proceedings. 2006;81:511-516.

6. Iconomou TG, Tsoutsos D, Frangia K, Gogas H, Papadopoulos S, Georgountzos V, et al. Malignant melanoma of the stomach presenting with an unknown primary lesion. Eur J Plast Surg. 2003;26:153-155.

7. Taal BG, Westerman H, Boot H, Rankin EM. Clinical and endoscopic features of melanoma metastases in the upper GI tract. Gastrointest Endosc. 1999;50(2):261263.

8. Nakahara H, Kitamura R, Shirasuna K. Simultaneous malignant melanoma and squamous cell carcinoma of the oral cavity: a case report. J Oral Maxillofac Surg. 1995;53:1455-1457.

9. Poggi SH, Madison JF, Hwu WJ, Bayar S, Salem RR. Colonic melanoma, primary or regressed primary. J Clin Gastroenterol. 2000;30:441-444.

10. Patel JK, Didolkar MS, Pickren JW, Moore RH. Metastatic pattern of malignant melanoma: a study of 216 autopsy cases. Am J Surg. 1978;135:807- 810.

11. Kamposioras K, Pentheroudakis G, Pectasides D, Pavlidis N. Malignant melanoma of unknown primary site. To make the long story short. A systematic review of the literature. Crit Rev Oncol Hematol. 2011;78:112126.

12. Vijuk G, Coates AS. Survival of patients with visceral metastatic melanoma from an occult primary lesion: A retrospective matched cohort study. Ann Oncol. 1998;9:419-422.

13. Ollila DW, Essner R, Wanek LA, Morton DL. Surgical resection for melanoma metastatic to the gastrointestinal tract. Arch Surg. 1996;131:975-980. 
14. Barth A, Wanek LA, Morton DL. Prognostic factors in 1,521 melanoma patients with distant metastases. J Am Coll Surg. 1995;181:193-201.

15. Kamposioras K, Pentheroudakis G, Pectasides D, Pavlidis N. Malignant melanoma of unknown primary site. To make the long story short. A systematic review of the literature. Crit Rev Oncol Hematol. 2011;78:112126.

16. Garbe C, Peris K, Hauschild A, Saiag P, Middleton M, Spatz A, et al. Diagnosis and treatment of melanoma: European consensus-based interdisciplinary guideline update 2012. Eur J Cancer. 2012;48:2375-2390. 